\title{
To Be or Not To Be: Technical and Cost Implications of Having a Confirmed Diagnosis of Lung Cancer (or not) Before Surgery
}

\section{Rudith Guzman}

Hospital Clínic de Barcelona

\section{Leandro Grando}

Hospital Clínic de Barcelona

David Sánchez-Lorente

Hospital Clínic de Barcelona

\section{Marc Boada}

Hospital Clínic de Barcelona

\section{Nestor Quiroga}

Hospital Clínic de Barcelona

\section{Pablo Paglialunga}

Hospital Clínic de Barcelona

\section{Laureano Molins}

Hospital Clínic de Barcelona

Angela Guirao ( $\sim$ guirao@clinic.cat)

Hospital Clínic de Barcelona

\author{
Alvar Agusti \\ Hospital Clínic de Barcelona \\ Carlos Guerrero \\ Hospital Clínic de Barcelona
}

\section{Research Article}

Keywords: Cost effectiveness, Lung cancer, Video-assisted thoracic surgery

Posted Date: February 23rd, 2022

DOI: https://doi.org/10.21203/rs.3.rs-1349099/v1

License: (c) (i) This work is licensed under a Creative Commons Attribution 4.0 International License.

Read Full License 


\section{Abstract}

Objective: To compare the costs and length of hospital stay between patients with a confirmed lung cancer (LC) diagnosis before surgery versus those without confirmed diagnosis.

Methods: Retrospective and single center study conducted on consecutive patients who underwent a LC surgical procedure, with or without a pathologically confirmed LC diagnosis before surgery, between March 2017 and December 2019. The main outcomes were costs and length of hospital stay (LOHS).

Results: Among the 269 screened patients, 254 met the inclusion/exclusion criteria and were included in the analysis, 196 (77.2\%) with confirmed LC diagnosis before surgery and 58 (22.8\%) without it. Unadjusted cost was significantly lower in patients who underwent surgery without histopathological diagnosis before surgery than in those who underwent surgery with a histopathological diagnosis before surgery (Hodges-Lehmann median difference: - $1,280.5 €$; 95\% confidence interval [Cl]: - 1,682.1 to -899.4€, $\mathrm{p}<0.0001)$. LOHS was significantly shorter in patients without a previous diagnosis of LC before surgery (4.0 [95\% Cl: 3.0 - 4.0] vs. 5.0 [95\% Cl: 5.0 - 6.0] days, p<0.0001). Median ( $95 \% \mathrm{Cl}$ ) LOHS was significantly lower in patients who underwent video-assisted thoracoscopy $(4.0$ [95\% $\mathrm{Cl} 4.0-4.0]$ days $)$ than in those who underwent open-surgery $(6.0[95 \% \mathrm{Cl} 5.0-6.0]$ days, $p<0.0001)$.

Conclusions: Patients who went into the operating-room without a previous confirmed diagnosis of LC resulted in lower direct costs, shorter LOHS, and increased use of VATS.

\section{Introduction}

Lung cancer (LC) is one of the cancers most often diagnosed (11.6\% of total cases) and the leading cause of cancer death in the world (18.4\% of the total cancer deaths $)^{1-3}$. Surgical removal of LC can cure it but, unfortunately, this is possible only in a minority of patients because most of them are diagnosed in advanced stages of the disease that precludes the surgical option ${ }^{4-8}$. Surgical treatment of LC is not only associated with better clinical outcomes, but it is also more cost-effective than other medical therapeutic alternatives ${ }^{9}$.

LC screening programs using low dose computed tomography (CT) of the thorax aim to diagnose LC in earlier clinical stages, so surgical removal (and cure) becomes possible. Two recent, large, randomized, controlled studies (NLST in the US, and NELSON in Europe) have demonstrated that LC screening programs with low-dose CT is associated with earlier LC detection and reduced all-cause mortality ${ }^{10,11 .}$ Yet, LC screening programs also identify a significant number of solitary pulmonary nodules (SPN) that may eventually be diagnosed of LC (or not) only after surgical removal ${ }^{12}$.

Whether the type of surgery required and/or associated cost vary when a diagnosis of LC has been established before surgery (or not) is unclear. This study sought to investigate these questions, which may become important as LC screening programs become widely deployed and implemented in the near future and the number of SPN without a confirmed LC diagnosis before surgery increase. 


\section{Methods}

\section{Study design, Patients, and Ethics}

Retrospective, single center analysis that compares the type of surgery, length of hospital stay (LOHS) and associated cost of patients who underwent a surgical procedure for LC in our institution between March 2017 and December 2019, with or without a pathologically confirmed LC diagnosis before surgery. The surgical indication was established by the multi-disciplinary LC Committee in our Institution. The study was approved by the Institutional Ethics Board of our institution "Comitè d'Ètica de la Investigació (CEIm)" (HCB/2020/0726).

\section{Study Variables}

We included in the analysis demographics, clinical variables, LC-related variables (LC type and stage, neoadjuvant therapy, if any), surgery-related variables (site, type of surgical approach (video-assisted (VATS) vs. open surgery), type of surgical procedure (wedge, segmentectomy, lobectomy, pneumonectomy), LOHS and estimated total cost per patient. The latter included the cost of the surgical procedure itself plus that of personnel, hospital stay, consumables, drugs, laboratory tests and other medical supplies but other potential costs, such as transport services, food services or other non-medical materials, were not included in the calculation.

\section{Statistical analysis}

Results are presented as number (percentage), mean \pm standard deviation and median [95\% confidence interval $(95 \% \mathrm{Cl})$ ], as appropriate. Data were tested for normal distribution using a D’Agostino-Pearson test. If data were normally distributed, a two-tailed independent-samples Student's t-test was used to compare groups, whereas when the distribution was not normal, the Mann-Whitney $U$ test was used instead. Categorical variables were compared using a Chi-square test and a Fisher's exact test, as needed. Analysis of covariance (ANCOVA) was used to contrast LOHS and costs between study groups. The model included "study group" as a factor and age, sex, smoking habit, TNM classification, tumor stage, FEV1, and performance access as covariates. A p value of less than 0.05 was considered significant.t. Analyses were performed using the MedCalc® Statistical Software version 20.014 (MedCalc Software Ltd, Ostend, Belgium) and the SPSS IBM Corp. Released 2019. IBM SPSS Statistics for Windows, Version 26.0. Armonk, NY: IBM Corp.

\section{Results}

\section{Patient characteristics}

During the study period, 269 patients underwent a surgical procedure for LC in our institution: 8 of them $(2.97 \%)$ had a benign lesion after pathologic analysis and baseline information was not complete in 7 
additional patients. These 15 patients were excluded from the current analysis, which finally included 254 patients with pathologically confirmed LC, $196(77.2 \%)$ of them before surgery and $58(22.8 \%)$ after it. Table 1 summarizes the main demographic and clinical characteristics of both groups. Age, sex, severity of airflow limitation and smoking history were similar. Adenocarcinoma was the most frequently diagnosed LC type in both groups, followed by squamous cell in patients with previous diagnosis and lepidic adenocarcinoma in patients without it (Table 2). Table 3 presents the distribution of LC stages in both groups. 
Table 1

Characteristics of patients with or without a previous diagnosis of LC before surgery.

\begin{tabular}{|c|c|c|c|}
\hline & WITH $(n=196)$ & WITHOUT $(n=58)$ & $P$ value \\
\hline Age, years & 68.0 (61.0 to 74.0$)$ & 70.0 (63.0 to 75.0$)$ & 0.2783 \\
\hline \multicolumn{4}{|l|}{ Median (IqR) } \\
\hline Sex, n (\%) & $72(36.7)$ & $15(25.9)$ & \multirow[t]{2}{*}{$0.1261^{b}$} \\
\hline Women & $124(63.3)$ & $43(74.1)$ & \\
\hline \multicolumn{4}{|l|}{ Men } \\
\hline $\mathrm{BMI}, \mathrm{Kg} / \mathrm{m}^{2}$ & 25.2 (23.2 to 27.7$)$ & Missing & N.A. \\
\hline \multicolumn{4}{|l|}{ Median (IqR) } \\
\hline Comorbidities, n (\%) & $93(47.4)$ & Missing & N.A. \\
\hline Yes & $103(52.6)$ & & \\
\hline \multicolumn{4}{|l|}{ No } \\
\hline Neoadjuvant therapy, n (\%) & 168 (92.3) & N.A. & N.A. \\
\hline None & $8(4.4)$ & & \\
\hline CT & $6(3.3)$ & & \\
\hline \multicolumn{4}{|l|}{$\mathrm{CT}+\mathrm{RT}$} \\
\hline Adjuvant therapy & $132(67.7)$ & N.A. & N.A. \\
\hline None & $37(19.0)$ & & \\
\hline CT & $26(13.3)$ & & \\
\hline \multicolumn{4}{|l|}{$\mathrm{CT}+\mathrm{RT}$} \\
\hline $\mathrm{FEV}_{1},(\%)$ & 83.0 (69.0 to 90.0$)$ & 83.0 (67.0 to 97.0$)$ & 0.5514 \\
\hline Median (IqR) & & & \\
\hline
\end{tabular}

IqR: Interquartile range; BMI: Body mass index; COPD: Chronic obstructive pulmonary disease; CT: Chemotherapy; RT: Radiotherapy; FEV 1 : Forced expiratory volume in 1 second; VATS: Video-assisted thoracoscopic surgery; LPS: Lobectomy procedure site; RUL: Right upper lobule; RML: Right middle lobule; RLL: Right lower lobule; LUL: Left upper lobule; LLL: Left lower lobule; NA: Not applicable. 


\begin{tabular}{|c|c|c|c|}
\hline & WITH $(n=196)$ & WITHOUT $(n=58)$ & $P$ value \\
\hline Smoking habit, n (\%) & $12(6.1)$ & $7(12.1)$ & $0.0525^{b}$ \\
\hline Never smoked & $53(27.0)$ & $22(37.9)$ & \\
\hline Current smoker & $122(62.2)$ & $29(50.0)$ & \\
\hline Past smoker & $9(4.6)$ & $0(0.0)$ & \\
\hline \multicolumn{4}{|l|}{ Unknown } \\
\hline Surgery approach, n (\%) & $106(54.1)$ & $58(100.0)$ & $<0.0001$ \\
\hline VATS & $90(45.9)$ & $0(0.0)$ & \\
\hline \multicolumn{4}{|l|}{ Open thoracotomy } \\
\hline LPS, n (\%) & $0(0.0)$ & $2(3.4)$ & $0.0459^{b}$ \\
\hline Culmen & $64(38.3)$ & $23(39.7)$ & \\
\hline RUL & $5(3.0)$ & $4(6.9)$ & \\
\hline RML & $34(20.4)$ & $11(19.0)$ & \\
\hline RLL & $44(26.3)$ & $8(13.8)$ & \\
\hline LUL & $20(12.0)$ & $10(17.2)$ & \\
\hline \multicolumn{4}{|l|}{ LLL } \\
\hline $\begin{array}{l}\text { IqR: Interquartile range; B } \\
\text { Chemotherapy; RT: Radic } \\
\text { thoracoscopic surgery; L } \\
\text { lobule; RLL: Right lower I }\end{array}$ & $\begin{array}{l}\text { ass index; COPD } \\
\mathrm{V}_{1} \text { : Forced expira } \\
\text { my procedure sit } \\
\text {-eft upper lobule }\end{array}$ & $\begin{array}{l}\text { obstructive pulmona } \\
\text { me in } 1 \text { second; VAT } \\
\text { ght upper lobule; RN } \\
\text { t lower lobule; NA: N }\end{array}$ & $\begin{array}{l}\text { ase; CT: } \\
\text { o-assisted } \\
\text { t middle } \\
\text { icable. }\end{array}$ \\
\hline
\end{tabular}


Table 2

Histopathological diagnosis in patients with or without a previous diagnosis of LC before surgery.

\begin{tabular}{|c|c|c|}
\hline & Diagnosis & $\mathbf{N}(\%)$ \\
\hline \multirow[t]{6}{*}{ WITH } & Adenocarcinoma & 95 (51.9) \\
\hline & Carcinoma in situ* & $10(5.5)$ \\
\hline & Large cells & $7(3.8)$ \\
\hline & Mixed & $6(3.8)$ \\
\hline & Squamous cells & $59(32.2)$ \\
\hline & Neuroendicrine & $6(3.3)$ \\
\hline \multirow[t]{7}{*}{ WITHOUT } & Adenocarcinoma & $23(39.7)$ \\
\hline & Lepidic adenocarcinoma & $20(34.5)$ \\
\hline & Atypical carcinoid & $3(5.2)$ \\
\hline & Typical carcinoid & $1(1.7)$ \\
\hline & Large cells & $2(3.4)$ \\
\hline & Small cells & $1(1.7)$ \\
\hline & Squamous cells & $8(13.8)$ \\
\hline
\end{tabular}


Table 3

Lung cancer stage, according to the 8th edition of the tumor, node and metastasis (TNM) classification system in patients with or without a previous diagnosis of LC before surgery.

\begin{tabular}{|llll|}
\hline TNM & WITH & WITHOUT & P value \\
\hline T1aN0M0 & $8(4.1)$ & $6(10.3)$ & $<0.0001$ \\
T1bN0M0 & $52(26.7)$ & $21(36.2)$ & \\
T1bN1M0 & $2(1.0)$ & $0(0.0)$ & \\
T1bN2M0 & $3(1.5)$ & $1(1.7)$ & \\
T1cN0M0 & $16(8.2)$ & $19(32.8)$ & \\
T1cN0M1c & $0(0.0)$ & $1(1.7)$ \\
T1cN1M0 & $4(2.0)$ & $0(0.0)$ \\
T2aN0M0 & $19(9.7)$ & $4(6.9)$ \\
T2aN1M0 & $3(1.5)$ & $1(1.7)$ \\
T2aN2M0 & $8(4.1)$ & $0(0.0)$ \\
T2bN0M0 & $12(6.0)$ & $3(5.1)$ \\
T2bN0M1b & $1(0.5)$ & $0(0.0)$ \\
T2bN1M0 & $4(2.0)$ & $0(0.0)$ \\
T2bN1M1b & $1(0.5)$ & $0(0.0)$ \\
T2N1M0 & $0(0.0)$ & $1(1.7)$ \\
T3N0M0 & $23(11.7)$ & $0(0.0)$ \\
T3N1M0 & $10(5.1)$ & $1(1.7)$ \\
T3N2M0 & $7(3.6)$ & $0(0.0)$ \\
T4N0M0 & $13(6.6)$ & $0(0.0)$ \\
T4N1M0 & $8(4.1)$ & $0(0.0)$ \\
T4N2M0 & $2(1.0)$ & $0(0.0)$ \\
\hline
\end{tabular}

\section{Surgical Access And Lohs}

Video-assisted thoracoscopic surgery (VATS) was used in all patients without a previous diagnosis of LC before surgery but only in $54.1 \%$ of those with a known one $(p<0.0001)$. Additionally, there were some 
differences in the lobectomy site between both groups $(p=0.05)($ Table 1$)$.

In the entire population studied, median LOHS in the population studied was significantly lower in patients who underwent VATS (4.0 [95\% Cl: 4.0-4.0] days) than in those who underwent open thoracotomy $(6.0$ [95\% Cl: $5.0-6.0]$ days, $p<0.0001)$ (Fig. 1A). Given that VATS was used in all patients without a previous diagnosis of LC before surgery, LOHS was significantly shorter in these patients (4.0 [3.0-4.0] vs. 5.0 [95\% Cl: 5.0-6.0] days, $\mathrm{p}<0.0001)$.

\section{Cost Analysis}

Figure 1B shows that, irrespective of having or not an established diagnosis of LC before surgery, median cost was lower in patients who underwent VATS $(2,915.3$ ([95\% Cl 2791.5-3285.3] $€)$ vs. open thoracotomy $(4,031.2$ [95\% Cl 3856.8-4359.1] $€$, (Hodges-Lehmann median difference: $-1,051.5 € ; 95 \% \mathrm{Cl}$ : $-1,428.5$ to $-693.9 €, p<0.0001)$. Differences persisted when analyzed in patients with a previous diagnosis of LC only (median difference, -657.9 [95\% Cl $-1,088.0$ to -137.1$] €, p<0.007$ ). This analysis could not be done in patients without a previous LC diagnosis because all of them underwent VATS.

Unadjusted cost was significantly lower in patients who underwent surgery without histopathological diagnosis before surgery (median cost, $2,440.6 € ; 95 \% \mathrm{Cl} 2,044.4$ to 2,706.4 $€$ ) than in those who underwent surgery with a histopathological diagnosis before surgery (Median 3,838 €; 95\% Cl: 3,438.6 to 4,042.8 $€$ ) (Hodges-Lehmann median difference: $-1,280.5 € ; 95 \% \mathrm{Cl}:-1,682.1$ to $-899.4 €, p<0.0001$ ). (Fig. 2).

After adjustment for covariates, there was a mean difference of $-1,437.2 €[95 \% \mathrm{Cl}:-2,010.8$ to $-863.7 €], p$ $<0.0001)$ ) cheaper in the group without previous diagnosis.

\section{Discussion}

This study shows that patients undergoing surgery because of LC without a previously histopathological established diagnosis (likely many of those potentially identified in LC screening programs) require less invasive surgical techniques (VATS), have a shorter LOHS and incur in lower costs than those with such a diagnosis before surgery.

We have previous reported that surgical treatment of LC is cheaper than medical management and has better outcomes ${ }^{9}$. Here, we extend these previous observations to investigate the impact of having (or not) a pathologically stablished diagnosis of LC before surgery. There is controversy on how to best approach the diagnosis of LC before surgery. Barnett et al ${ }^{13}$ suggested that in patients with SPN, presurgical CT guide percutaneous lung biopsy was the most cost-effective strategy. Yet, this assumed that rates of resection of nonmalignant lung nodules are high ${ }^{14}$. On average, approximately $25 \%$ of the thoracic surgical procedures performed during the various randomized controlled LC screening trials were done for benign nodules ${ }^{15}$. By contrast, in keeping with our findings here, Cho et al found that surgery 
resection without previous tissue histopathological diagnosis was more cost-effective and reduced the length of hospital stay in nodular ground-glass opacities with high suspicious of malignancy ${ }^{16}$.

The main observation of this study is that not having an established LC diagnosis before surgery is associated with less invasive surgical procedures (VATS), shorter LOHS and reduced costs. VATS is increasingly used in thoracic surgery and many observational studies have shown that it has at least the same long-term outcome benefits in the treatment of LC as the traditional open thoracotomy ${ }^{17,18}$. VATS lobectomy for patients with early-stage LC is a standard surgical approach and is associated with lower morbidity and improved survival rates compared with thoracotomy ${ }^{19,20}$. Further, VATS is potentially more cost-effective than thoracotomy ${ }^{21,22}$. Thus, we speculate that the reduced cost observed in our study is likely related to a higher use of VATS and a reduced LOHS.

Our study has two potential limitations. First, it is a retrospective analysis. Second, we considered only direct medical costs, whereas indirect costs such as transport services, food services, other non-medical materials, and working time lost were not taken into consideration.

In conclusion, our study indicates that having a previously established diagnosis of LC in patients with a suspicious SPN before surgery is not a necessary pre-requisite and results in shorter LOHS, increased use of VATS and reduced direct costs.

\section{Declarations}

\section{Availability of materials and data}

The datasets generated during and/or analyzed during the current study are available from the corresponding author on reasonable request.

\section{Acknowledgements}

Editorial assistance in the preparation of this article was provided by Antonio Martinez (MD) of Ciencia y Deporte Itd.

\section{Author Contribution}

All authors met the ICMJE authorship criteria. All authors made substantial contributions to conception, design, analysis and interpretation of data, contributed to writing the article, provided critical revision of the manuscript, and approved the final version.

\section{Disclosure of potential conflicts of interest}

None of the authors have any conflict of interest to declare.

\section{Ethics Declaration}


"All procedures performed in studies involving human participants were in accordance with the ethical standards of the institutional and/or national research committee and with the 1964 Helsinki declaration and its later amendments or comparable ethical standards. The local ethics committee waived the need for written informed consent of the participants for the study".

\section{Ethics approval}

This study was approved by the local ethics committees and was performed with the principles of the Declaration of Helsinki.

\section{References}

1. Malvezzi, M., et al. European cancer mortality predictions for the year 2017, with focus on lung cancer. Ann Oncol 28(5):1117-1123 (2017).

2. Bray, F., et al. Global cancer statistics 2018: GLOBOCAN estimates of incidence and mortality worldwide for 36 cancers in 185 countries. CA Cancer J Clin 68(6):394-424 (2018).

3. Babar, L., Modi, P., Anjum, F. Lung Cancer Screening. 2020 Dec 4. In: StatPearls[Internet]. Treasure Island (FL): StatPearls Publishing; 2021 Jan. Available in:

https://www.ncbi.nlm.nih.gov/books/NBK537283/ Last accessed October 29, 2021.

4. Asamura, H., Aokage, K., Yotsukura, M. Wedge Resection Versus Anatomic Resection: Extent of Surgical Resection for Stage I and II Lung Cancer. Am Soc Clin Oncol Educ Book 37:426-433 (2017).

5. Pérez-Martínez, O., Vidal-García, I., Montero-Martínez, C., Provencio, M., Ruano-Ravina, A. Description and Survival of Stage I and II Lung Cancer Patients. Arch Bronconeumol 54(8):420-426 (2018).

6. Waller, D. A. Surgical management of lung cancer with multiple lesions: implication of the new recommendations of the $8<$ sup $>$ th $<$ / sup $>$ edition of the TNM classification for lung cancer. $J$ Thorac Dis (Suppl 22): S2686-S2691 (2018).

7. Rosen, J. E., et al. The Natural History of Operable Non-Small Cell Lung Cancer in the National Cancer Database. Ann Thorac Surg 101(5):1850-1855 (2016).

8. Postmus, P. E., et al; ESMO Guidelines Committee. Early and locally advanced non-small-cell lung cancer (NSCLC): ESMO Clinical Practice Guidelines for diagnosis, treatment and follow-up. Ann Oncol 28(suppl_4):iv1-iv21 (2017).

9. Guzman, R., et al. Outcomes and cost of lung cancer patients treated surgically or medically in Catalunya: cost-benefit implications for lung cancer screening programs. Eur $\mathrm{J}$ Cancer Prev 29(6):486-492 (2020).

10. National Lung Screening Trial Research Team, Aberle, D. R., et al. Reduced lung-cancer mortality with low-dose computed tomographic screening. N Engl J Medn365(5):395-409 (2011).

11. de Koning, H. J., et al. Reduced Lung-Cancer Mortality with Volume CT Screening in a Randomized Trial. N Engl J Med 382(6):503-513 (2020). 
12. Gould, M. K., et al. Recent Trends in the Identification of Incidental Pulmonary Nodules. Am J Respir Crit Care Med 192(10):1208-1214 (2015).

13. Barnett, J., et al. Pre-surgical lung biopsy in management of solitary pulmonary nodules: a cost effectiveness analysis. J Med Econ 22(12):1307-1311 (2019).

14. Barta, J. A., et al. Lung Cancer Diagnosis by Fine Needle Aspiration Is Associated With Reduction in Resection of Nonmalignant Lung Nodules. Ann Thorac Surg 103(6):1795-1801 (2017).

15. Detterbeck, F. C., Mazzone, P. J., Naidich, D. P., Bach, P. B. Screening for lung cancer:Diagnosis and management of lung cancer, 3rd ed: American College of Chest Physicians evidence-based clinical practice guidelines. Chest 143(5 Suppl):e78S-e92S (2013).

16. Cho, J., et al. Surgical resection of nodular ground-glass opacities without percutaneous needle aspiration or biopsy. BMC Cancer 14:838 (2014).

17. Van Schil, P. E., et al.nSurgical treatment of early-stage non-small-cell lung cancer. EJC Suppl 11(2):110-122 (2013).

18. Detterbeck, F., Molins, L. Video-assisted thoracic surgery and open chest surgery in lung cancer treatment: present and future. J Vis Surg 2:173 (2016).

19. Whitson, B. A., Groth, S. S., Duval, S. J., Swanson, S. J., Maddaus, M. A. Surgery for early-stage nonsmall cell lung cancer: a systematic review of the video-assisted thoracoscopic surgery versus thoracotomy approaches to lobectomy. Ann Thorac Surg 86(6):2008-2016; discussion 2016-2018 (2008).

20. Dziedzic, R., Marjański, T., Rzyman, W. A narrative review of invasive diagnostics and treatment of early lung cancer. Transl Lung Cancer Res 10(2):1110-1123 (2021).

21. Fang, H. Y., et al. Cost and effectiveness of video-assisted thoracoscopic surgery for clinical stage I non-small cell lung cancer: a population-based analysis. J Thorac Dis 6(12):1690-1696 (2014).

22. Bendixen, M., Kronborg, C., Jørgensen, O. D., Andersen, C., Licht, P. B. Cost-utility analysis of minimally invasive surgery for lung cancer: a randomized controlled trial. Eur J Cardiothorac Surg 56(4):754761 (2019).

\section{Figures}



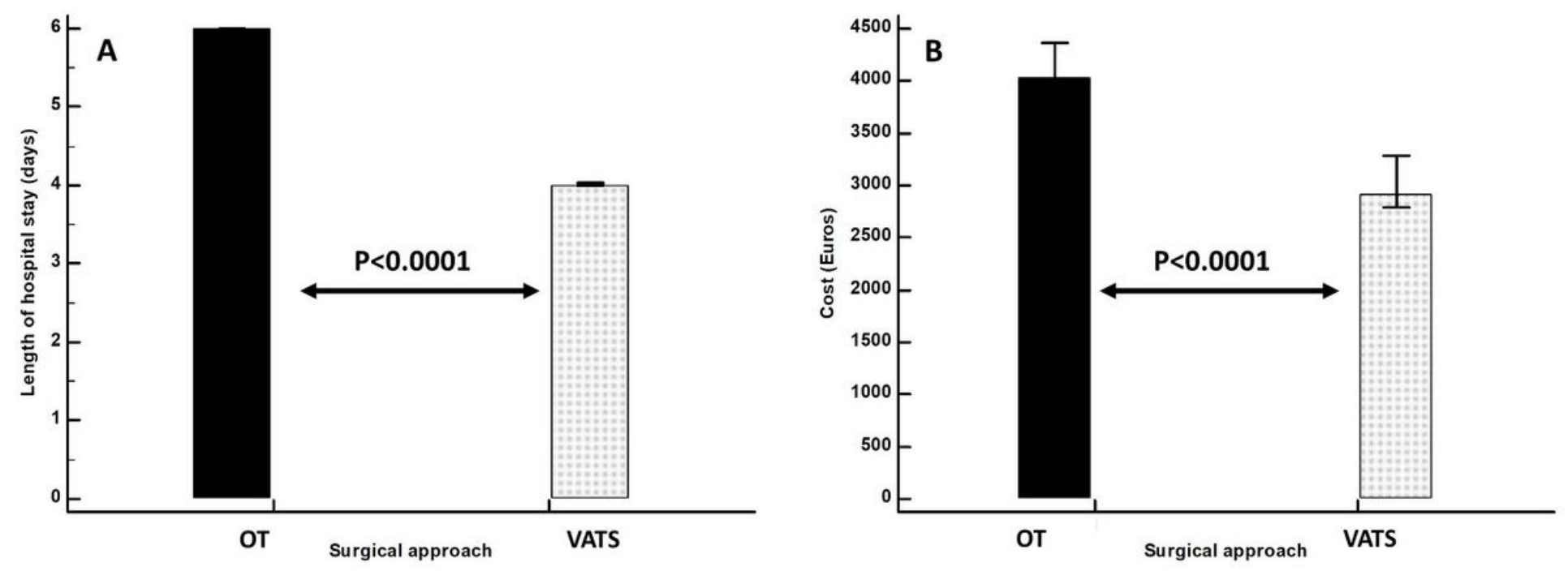

\section{Figure 1}

A comparison of the length of hospital stay (Panel A) and median costs (Panel B) between lung-cancer patients who underwent Video-assisted thoracoscopic surgery (VATS) and those who did it open thoracotomy (OT), irrespective of having or not an established diagnosis of lung cancer before surgery. Statistical significance was determined using the Mann-Whitney U test.

Vertical bars represent $95 \%$ confidence interval of the median.

A. Hodges-Lehmann median difference: -1.0 day; $95 \% \mathrm{Cl}:-2.0$ to -1.0 days, $p<0.0001$.

B. Hodges-Lehmann median difference: $-1,051.5 € ; 95 \% \mathrm{Cl}:-1428.5$ to $-693.9 €, \mathrm{p}<0.0001$. 


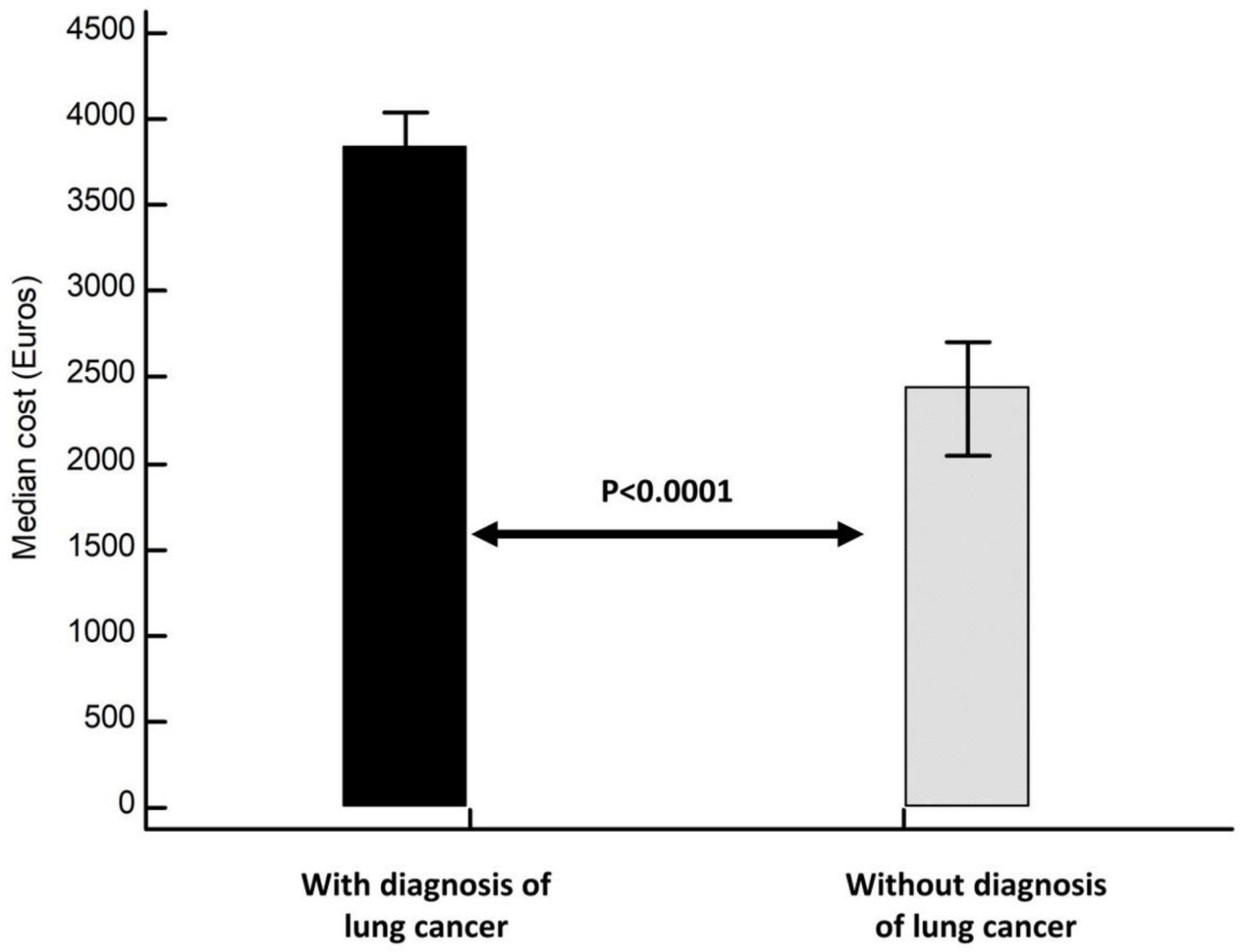

Figure 2

A comparison of the median cost between lung-cancer patients who underwent surgy with a histopathological diagnosis before surgery (Group I) and those who did it after surgery (Group II). Statistical significance was determined using the Mann-Whitney U test.

Vertical bars represent $95 \%$ confidence interval of the median.

Hodges-Lehmann median difference: $-1,280.5 € ; 95 \% \mathrm{Cl}:-1,682.1$ to $-899.4 €, \mathrm{p}<0.0001$. 